\title{
Search for excited states in ${ }^{3} \mathrm{H}$ and ${ }^{3} \mathrm{He}$
}

\author{
Attila Csótó ${ }^{1,2}$ and G. M. Hale ${ }^{1}$ \\ ${ }^{1}$ Theoretical Division, Los Alamos National Laboratory, Los Alamos, New Mexico 87545 \\ ${ }^{2}$ Department of Atomic Physics, Eötvös University, Puskin u. 5-7, H-1088 Budapest, Hungary
}

(August 26, 1998)

\begin{abstract}
The $d+N$ systems are studied in a three-body model, using phenomenological $\mathrm{N}-\mathrm{N}$ interactions. The scattering matrices are calculated by using the Kohn-Hulthén variational method. Then, they are analytically continued to complex energies and their singularities are localized. We find a virtual state at $E=-1.66 \mathrm{MeV}$ in ${ }^{3} \mathrm{H}$ and a pair of states at $E=(-0.42 \pm i 0.52) \mathrm{MeV}$ in ${ }^{3} \mathrm{He}$ relative to the $d+N$ thresholds, respectively. In addition, we discuss some general aspects and problems of virtual states which may be useful also in the study of other systems, like ${ }^{10} \mathrm{Li}$.

PACS number(s): 21.45.+v, 24.30.Gd, 27.10.+h
\end{abstract}

\section{INTRODUCTION}

The lightest nucleus with a well-established spectrum of excited states is ${ }^{4} \mathrm{He}$. There have been numerous attempts, both experimental and theoretical, to identify excited states in $A=3$ nuclei; for an extensive review see Ref. [1]. Although there are indications for resonance-like behavior in the $3 n$ and $3 p$ systems in certain experiments [2], others do not seem to support these findings [3]. Theoretically the most well-established case is a broad threeneutron (and three-proton) resonance in the $J^{\pi}=3 / 2^{+}$ partial wave [4. However, the existence of these structures has not been confirmed by experiments yet.

According to Ref. [1], no unambiguous experimental evidence has been found to support the existence of lowlying ${ }^{3} \mathrm{H}$ and ${ }^{3} \mathrm{He}$ resonances. However, several calculations, mostly confined to small model spaces, indicate a virtual state in ${ }^{3} \mathrm{H}$, and corresponding subthreshold resonances in ${ }^{3} \mathrm{He}$. Recently, the authors of a $\mathrm{H}\left({ }^{6} \mathrm{He},{ }^{4} \mathrm{He}\right)$ experiment have claimed to find an excited state of ${ }^{3} \mathrm{H}$ at $E^{*}=7 \pm 0.3 \mathrm{MeV}$ excitation energy with $\Gamma=0.6 \pm 0.3$ $\mathrm{MeV}$ width [5]. According to one interpretation [6] this is a $1 / 2^{+}$excited state formed mainly in the $(n n) p$ channel. In the mirror ${ }^{3} \mathrm{He}$ nucleus the Ref. [5] experiment does not indicate an excited state. However, in a recent work [7] it was shown that the effective range function of the doublet $d+p$ scattering has a singularity at a negative energy, just below the $d+p$ threshold. This would indicate the existence of subthreshold virtual states in ${ }^{3} \mathrm{He}$.

In the present work we study the ${ }^{3} \mathrm{H}$ and ${ }^{3} \mathrm{He}$ nuclei with the aim to clarify some of the possibilities mentioned above. We describe the $d+N$ scattering in a nonrelativistic three-body potential model and study the analytic properties of the scattering matrices. Since virtual states play an important role in our study, we briefly discuss some of their features in Sec. II, and show some problems associated with their experimental investigation. Our three-body calculations are reviewed in Sec. III, while Sec. IV offers some conclusions.

\section{VIRTUAL STATES IN SCATTERING THEORY}

The spectrum of a quantum scattering system generally consists of bound states and continuum states. The continuum has structure caused by the complex-energy singularities of the scattering matrix. For a broad class of physically interesting interactions all singularities within the meromorphic region of the potential are physical and correspond to solutions of the Schrödinger equation with purely outgoing asymptotics. The following types of singularities are usually defined on the complex $k$ (wave number) plane: i) bound states with $k_{B}=i \gamma(\gamma>0)$; ii) virtual (antibound) states with $k_{A}=-i \gamma(\gamma>0)$; resonances with $k_{D}= \pm \kappa-i \gamma(\kappa, \gamma>0 ; \kappa>\gamma)$; and quasi-resonances with $k_{C}= \pm \kappa-i \gamma(\kappa, \gamma>0 ; \kappa<\gamma)$.

Our main interest in the present work is in the virtual states. Interestingly, although a virtual state is an unbound state with exponentially increasing wave function, it corresponds to negative energy, $E_{V}=k^{2} \hbar^{2} / 2 m=$ $-\gamma^{2} \hbar^{2} / 2 m$. The cross section of a process which involves a virtual state behaves like $\sigma(E) \sim 1 /\left(E+\left|E_{V}\right|\right)$ for $E>0$. Thus it is singular at the unphysical negative energy $E=-\left|E_{V}\right|$, and increases with decreasing positive energies. This behavior can have rather strange effects on experimental measurements. If a measurement is performed on a system which has a virtual state and the interaction-free cross section (phase space) of the measured process drops to zero with decreasing energy, then the result of the measurement is a cross section with a low-energy peak. This may be interpreted as a lowenergy resonance at positive energies. However, the peak cannot be fitted with a Breit-Wigner form, which causes the extracted "resonance parameters" to be highly dependent on the fit procedure. A good example of this experimental difficulty concerning virtual states is ${ }^{10} \mathrm{Li}$. We believe that the experimental confusion regarding the nature of the ground state of ${ }^{10} \mathrm{Li}[8]$ is caused by the fact that there is a virtual state in the ${ }^{9} \mathrm{Li}+n$ system [9]. The energy of this state is negative, for instance $E_{V} \approx-0.03$ $\mathrm{MeV}$ for the P2 interaction of Ref. [9]. If, however, the experimental procedures assume a low-energy resonance 
in this system and try to fit the various measured cross sections accordingly by Breit-Wigner forms, then they can get rather different results.

We would like to emphasize that pure virtual states with pure imaginary wave number can only exist in neutral systems with two-body asymptotics and only in $S$ wave states. By making the interaction more attractive the virtual state can be moved from the negative imaginary $k$-axis to the positive one, ending up with a bound state [10]. If, however, a Coulomb or centrifugal barrier is added to the interaction, then the virtual pole moves from the imaginary axis into the complex $k$ plane resulting in a quasi-resonance. In order to illustrate this process we show in Fig. 11 the pole trajectory corresponding to the ${ }^{1} S_{0} N+N$ system. We start from $n+n$ and smoothly switch on the $p+p$ Coulomb interaction to finally end up as the $p+p$ system. One can see that the effect of the Coulomb force is to move the virtual pole into the complex plane (and to create a conjugate pole) as discussed above. We use here the Eikemeier-Hackenbroich (EH) N-N interaction [11]. The $n+n$ and $p+p$ poles are at $E_{n n}=-0.134$ $\mathrm{MeV}$ and $E_{p p}=(-0.101 \pm i 0.515) \mathrm{MeV}$ energies respectively. These numbers are in reasonable agreement with those that can be extracted from experimental data, $E_{n n}=-0.123 \mathrm{MeV}$ and $E_{p p}=(-0.140 \pm i 0.467) \mathrm{MeV}$ [12]. Our interaction is charge-independent, so it gives the same pole position for $n+n$ and $n+p$. Naturally, it cannot reproduce the experimental $E_{n p}=-0.066 \mathrm{MeV}$ [12].

We note that although one cannot have pure virtual poles in charged systems, a pair of quasi-resonance poles close to the negative imaginary $k$ axis can have observable effects at positive real energies, like in the case of $p+p$. The reason for this is the fact that unlike in the case of usual resonance poles, both of such poles are roughly the same distance from the real energy axis of the physical energy sheet. This is not the case for usual resonant states. The $\varepsilon=E_{r}-i \Gamma / 2\left(E_{r}, \Gamma>0\right)$ main pole of a usual resonance can be reached by simply crossing the real energy axis and going to the fourth quadrant of the energy plane. However, the conjugate pole can only be reached through a long path by crossing the real energy axis, going into the fourth quadrant, and then encircling the origin and ending up at $\varepsilon=E_{r}+i \Gamma / 2\left(E_{r}, \Gamma>0\right)$.

To recap our brief discussion of virtual states, we emphasize the sometimes forgotten fact that the energy corresponding to these states is negative. We also note that in charged systems pure virtual states cannot exist. However, the effect of a pair of conjugate quasi-resonances close to the negative imaginary $k$ axis (corresponding to a virtual state in a neutral system) can have significant observable effects.

\section{THREE-BODY CALCULATIONS FOR $d+\mathrm{N}$ SCATTERING}

We solve the three-body Schrödinger equation for ${ }^{3} \mathrm{H}$ and ${ }^{3} \mathrm{He}$ using the $\mathrm{EH} \mathrm{N}-\mathrm{N}$ interaction. This interaction gives a good general description of the $N+N$ scattering data and the deuteron properties [11]. Technical problems prevent us from using the most modern interactions, but for our current purposes the $\mathrm{EH}$ interaction is quite satisfactory. In our calculations the same nucleon mass $M_{N}=\left(M_{p}+M_{n}\right) / 2$ is used for both protons and neutrons.

In this paper we study only $J^{\pi}=1 / 2^{+}$states. As a first step we solve the bound-state problem of ${ }^{3} \mathrm{H}$ and ${ }^{3} \mathrm{He}$ using the variational Gaussian-basis coupled rearrangement channel method [13]. We include all 23 channels with $l_{1}, l_{2} \leq 2$ in the $\left[\left[\left(S_{1}, S_{2}\right) S_{12}, S_{3}\right] S,\left(l_{1}, l_{2}\right) L\right] J^{\pi}$ coupling scheme. Here $S_{1}=S_{2}=S_{3}=1 / 2$ are the nucleon spins, $S_{12}$ is the coupled two-nucleon intrinsic spin, $S$ is the the total intrinsic spin, $l_{1}$ and $l_{2}$ are the orbital angular momenta of the two relative motions, respectively, $L$ is the total orbital angular momentum, $J$ is the total spin, and $\pi=(-1)^{l_{1}+l_{2}}$ is the parity. Our calculated binding energies, $E_{3} \mathrm{H}=-7.65 \mathrm{MeV}$ and $E_{3 \mathrm{He}}=-6.99 \mathrm{MeV}$, are smaller than the experimental values $E_{3 \mathrm{H}}^{\text {Exp }}=-8.482 \mathrm{MeV}$ and $E_{3 \mathrm{He}}^{\text {Exp }}=-7.718 \mathrm{MeV}$, respectively. Our triton binding energy is close to those coming from calculations that use the most modern twonucleon forces [14]. Thus, a large part of the underbinding can be attributed to the lack of three-body forces in our model.

We find that by keeping only the 9 most important channels, shown in Table If, the binding energies change by only $0.05 \mathrm{MeV}$. In the following we keep only these 9 channels. In order to have a coupled ${ }^{3} S_{1}-{ }^{3} D_{1}$ deuteron in our asymptotic $d+N$-type wave function components, we use the $\left[\left[\left[\left(S_{1}, S_{2}\right) S_{12}, l_{1}\right] I_{1}, S_{3}\right] I, l_{2}\right] J^{\pi}$ coupling scheme in the scattering calculations. Here $I_{1}$ is the total (intrinsic plus orbital) spin of the two-nucleon subsystem and $I$ comes from the coupling of $I_{1}$ and $S_{3}$. We show our model space in this coupling in Table III. Hence, we have 7 channels: two channels containing a ${ }^{3} S_{1}-{ }^{3} D_{1}$ deuteron plus a nucleon with $l_{2}=0$ and 2 , respectively (lines $1-2$ and $3-4$ in Table II), three channels with ${ }^{3} D$ states inside the $n+p$ system (lines 5,6 , and 7 in Table II), and two channels with ${ }^{1} S_{0} n+p$ and $n+n\left(p+p\right.$ for $\left.{ }^{3} \mathrm{He}\right)$ two-body subsystems, respectively (lines 8 and 9 in Table II). The multichannel scattering problem is solved by using the Kohn-Hulthén variational method for the $S$ matrix 15. In all our calculations we remain below the three-body breakup threshold. It means that in channels $5-9$ in Table II the wave functions have three-body bound-state asymptotics [16]. In these channels we keep the same Gaussian basis as in the bound state calculations. 
The scattering matrices, coming from the KohnHulthén calculations, are analytically continued to the multisheeted complex Riemann energy surface using the methods of Ref. [17], and their singularities are localized. As a test calculation we searched for the boundstate poles and found them at the same energies as in the bound-state calculations. In addition, a virtual state is found in ${ }^{3} \mathrm{H}$ at $E_{V}^{3} \mathrm{H}=-1.66 \mathrm{MeV}$, relative to the $d+n$ threshold. We note that as our triton ground state is underbound so is probably this virtual state. The effect of underbinding in a virtual state is that $\left|E_{V}\right|$ becomes larger 10. This means that if our $N-N$ interaction reproduced the correct binding energy of ${ }^{3} \mathrm{H}$ and ${ }^{3} \mathrm{He}$ then the virtual-state pole would be closer to zero energy. In ${ }^{3} \mathrm{He}$ we find a pair of conjugate poles at $E_{V}^{3} \mathrm{He}=(-0.42 \pm i 0.52) \mathrm{MeV}$, relative to the $d+p$ threshold. The insufficient attraction in our model probably moves the poles too far away from the negative energy axis.

A practical way to extract the parameters of the virtual states of ${ }^{3} \mathrm{H}$ and ${ }^{3} \mathrm{He}$ from experimental data is to describe these nuclei within the extended $R$-matrix model 18], which can be continued to complex energies. An extensive analysis of the $A=3$ data is being performed using this method 19]. Preliminary results indicate the presence of ${ }^{2} S_{1 / 2}{ }^{3} \mathrm{H}$ and ${ }^{3} \mathrm{He}$ virtual states at $E_{V}^{3} \mathrm{H}=-1.07 \mathrm{MeV}$ and $E_{V}^{3} \mathrm{He}=(-0.72 \pm i 0.23) \mathrm{MeV}$, respectively. The results of our three-body calculations are consistent with these findings if we take into account the fact that the insufficient binding probably increases $\left|E_{V}^{3} \mathrm{H}\right|$ and pushes the ${ }^{3} \mathrm{He}$ pole away from the negative axis.

In comparing our results to previous calculations, we note that in Ref. [20], negative-energy poles were found in the ${ }^{2} S_{1 / 2} k \cot \delta$ function for $d+n$ at $\approx-160 \mathrm{keV}$ and for $d+p$ at $\approx-25 \mathrm{keV}$, using the $S$-wave MT IIII interaction of Ref. [21]. A similar pole was found in the $d+p$ phase-shift analysis of Black et al. [22]. These real-energy poles in the $d+N$ effective-range expansion are clearly related to the presence of the virtual states we have found, as will be discussed in a later publication [19.

\section{CONCLUSION}

In summary, we have localized virtual $d+N$ states in ${ }^{3} \mathrm{H}$ and ${ }^{3} \mathrm{He}$ in a model that contains all relevant threebody channels. These states can be considered as special excited states of the $A=3$ nuclei. Our results undoubtedly show the existence of these structures, although their properties could change somewhat if the most modern N-N interactions with three-body forces were used. Such calculations are, however, beyond the scope of the present work.

\section{ACKNOWLEDGMENTS}

This work was performed under the auspices of the U.S. Department of Energy. Some of the calculations were performed at the Physics Department of Aarhus University, Denmark. A. C. thanks K. Langanke for his help and the Danish Research Council and the Theoretical Astrophysics Center for partial financial support. This work was supported by OTKA Grant. F019701 and by the Bolyai Fellowship of the Hungarian Academy of Sciences.

[1] K. Möller and Yu. V. Orlov, Sov. J. Part. Nucl. 20, 569 (1989).

[2] J. Sperinde, D. Frederickson, R. Hinkins, V. PerezMendez, and B. Smith, Phys. Lett. 32B, 185 (1970); L. E. Williams, C. J. Batty, B. E. Bonner, C. Tschalär, H. C. Benöhr, and A. S. Clough, Phys. Rev. Lett. 23, 1181 (1969); A. Stetz et al., Nucl. Phys. A457, 669 (1986).

[3] M. Yuly et al., Phys. Rev. C 55, 1848 (1997); M. Palarczyk et al., Phys. Rev. C 58, 645 (1998).

[4] A. Csótó, H. Oberhummer, and R. Pichler, Phys. Rev. C 53, 1589 (1996).

[5] D. V. Aleksandrov, E. Yu. Nikol'skii, B. G. Novatskii, and D. N. Stepanov, JETP Lett. 59, 320 (1994)

[6] A. L. Barabanov, JETP Lett. 61, 7 (1995).

[7] A. Kievsky, S. Rosati, M. Viviani, C. R. Brune, H. J. Karwowski, E. J. Ludwig, and M. H. Wood, Phys. Lett. B 406, 292 (1997).

[8] W. Benenson, Nucl. Phys. A588, 11c (1995).

[9] I. J. Thompson and M. V. Zhukov, Phys. Rev. C 49, 1904 (1994).

[10] W. Glöckle, The Quantum Mechanical Few-Body Problem (Springer-Verlag Berlin Heidelberg, 1983) pp. 27-33.

[11] H. Eikemeier and H. H. Hackenbroich, Nucl. Phys. A169, 407 (1971).

[12] L. P. Kok, Phys. Rev. Lett. 45, 427 (1980).

[13] M. Kamimura, Phys. Rev. A 38, 621 (1988); H. Kameyama, M. Kamimura, and Y. Fukushima, Phys. Rev. C 40, 974 (1989).

[14] A. Nogga, D. Hüber, H. Kamada, and W. Glöckle, Phys. Lett. B409, 19 (1997).

[15] M. Kamimura, Prog. Theor. Phys. Suppl. 62, 236 (1977).

[16] S. P. Merkur'ev, Yad. Fiz. 19, 447 (1974) [Sov. J. Nucl. Phys. 19, 222 (1974)].

[17] A. Csótó, R. G. Lovas, and A. T. Kruppa, Phys. Rev. Lett. 70, 1389 (1993); A. Csótó and G. M. Hale, Phys. Rev. C 55, 536 (1997).

[18] G. M. Hale, R. E. Brown, and N. Jarmie, Phys. Rev. Lett. 59, 763 (1987).

[19] G. M. Hale and A. Csótó, to be published.

[20] C. R. Chen, G. L. Payne, J. L. Friar, and B. F. Gibson, Phys. Rev. C 39, 1261 (1989). 
[21] R. A. Malfliet and J. A. Tjon, Nucl. Phys. A127, 161 (1969).

[22] T. C. Black, H. J. Karwowski, E. J. Ludwig, A. Kievsky, S. Rosati, and M. Viviani, Nucl. Phys. A631, 680c (1998).

TABLE I. Channel configurations used in our 9-channel calculations in the $\left[\left[\left(S_{1}, S_{2}\right) S_{12}, S_{3}\right] S,\left(l_{1}, l_{2}\right) L\right] J^{\pi}$ coupling scheme.

\begin{tabular}{cccccc}
\hline \hline Configuration & $S_{12}$ & $S$ & $l_{1}$ & $l_{2}$ & $L$ \\
\hline$N(p n)$ & 1 & $1 / 2$ & 0 & 0 & 0 \\
$N(p n)$ & 1 & $3 / 2$ & 2 & 0 & 2 \\
$N(p n)$ & 1 & $3 / 2$ & 0 & 2 & 2 \\
$N(p n)$ & 1 & $1 / 2$ & 2 & 2 & 0 \\
$N(p n)$ & 1 & $1 / 2$ & 2 & 2 & 1 \\
$N(p n)$ & 1 & $3 / 2$ & 2 & 2 & 1 \\
$N(p n)$ & 1 & $3 / 2$ & 2 & 2 & 2 \\
$N(p n)$ & 0 & $1 / 2$ & 0 & 0 & 0 \\
$p(n n)$ or $n(p p)$ & 0 & $1 / 2$ & 0 & 0 & 0 \\
\hline \hline
\end{tabular}

TABLE II. Channel configurations used in our 9-channel calculations in the $\left[\left[\left[\left(S_{1}, S_{2}\right) S_{12}, l_{1}\right] I_{1}, S_{3}\right] I, l_{2}\right] J^{\pi}$ coupling scheme.

\begin{tabular}{cccccc}
\hline \hline Configuration & $S_{12}$ & $l_{1}$ & $I_{1}$ & $I$ & $l_{2}$ \\
\hline$N(p n)$ & 1 & 0 & 1 & $1 / 2$ & 0 \\
$N(p n)$ & 1 & 2 & 1 & $1 / 2$ & 0 \\
$N(p n)$ & 1 & 0 & 1 & $3 / 2$ & 2 \\
$N(p n)$ & 1 & 2 & 1 & $3 / 2$ & 2 \\
$N(p n)$ & 1 & 2 & 2 & $3 / 2$ & 2 \\
$N(p n)$ & 1 & 2 & 2 & $5 / 2$ & 2 \\
$N(p n)$ & 1 & 2 & 3 & $5 / 2$ & 2 \\
$N(p n)$ & 0 & 0 & 0 & $1 / 2$ & 0 \\
$p(n n)$ or $n(p p)$ & 0 & 0 & 0 & $1 / 2$ & 0 \\
\hline \hline
\end{tabular}

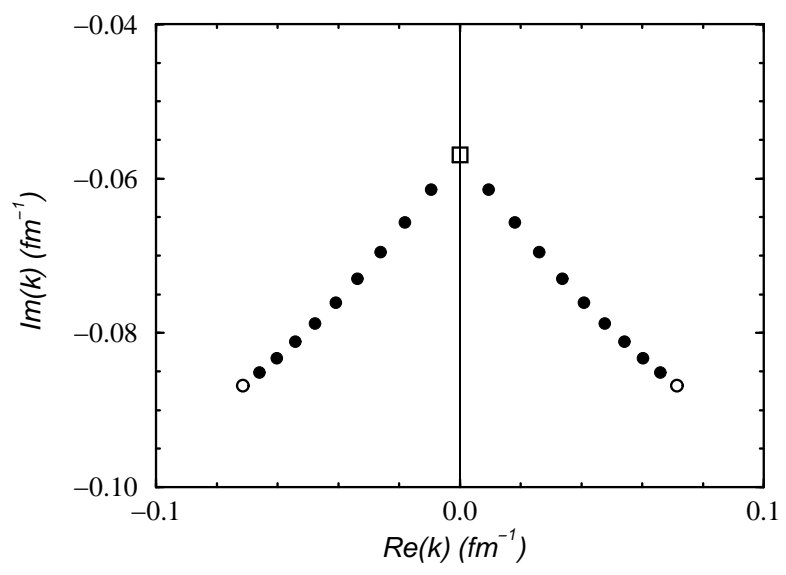

FIG. 1. Trajectories of the ${ }^{1} S_{0} N+N S$-matrix poles. The open square corresponds to the $n+n$ and $n+p$ poles, wile the open circles denote the pair of conjugate poles in the $p+p$ system. The filled circles come from calculations where $c \cdot V_{\text {Coul }}^{p p}$ is added to the $n+n$ interaction $(0<c<1)$. 